\title{
Morphological basis of clinical Hepatology
}

\begin{abstract}
Morphological organization of the liver in humans normally studied at a sufficiently high level. The functions of the liver, which play an important role in the regulation of metabolic and adaptive processes was researched in detail, but the dynamics of morphological and functional changes in the liver in different diseases isn't studied enough. However, many diseases are accompanied by clinical symptoms that may be due to the lack of functional activity of the liver.

In the modern world, there is a steady growth of both primary liver diseases and secondary liver lesions in diseases of other organs and systems. Detailed knowledge of both microanatomy and liver microanatomy, by practitioners and, in particular, by surgeons, contribute to the objectification of the choice of treatment tactics and, accordingly, to improve the results of patient treatment with liver diseases.
\end{abstract}

Keywords: liver microanatomy, liver microanatomy, liver diseases
Volume 10 Issue 4 - 2019

Milyukov VE, Sharifova HM, Sharifor ER

Department of Human Anatomy, First Moscow State Medical University, Russia

Correspondence: Milyukov VE, Department of Human Anatomy, First Moscow State Medical University Moscow, Russia, Emailmilyucov@mail.ru

Received: June 24, 2019 | Published: August 13, 2019
Abbreviations: $\mathrm{CH}$, chronic viral hepatitis; $\mathrm{AIO}$, acute intestinal obstruction; SPN, sensory peptidergic nerve fibers; WHO, world health organization

\section{Liver diseases}

Liver diseases and liver damage, which are complications of diseases of the gastrointestinal tract, as well as other organs and systems, which manifest as liver failure, remain a significant problem of modern practical medicine throughout the world.

Viral hepatitis is the most common cause of liver failure. According to the WHO, more than 2 billion people are infected with the hepatitis $\mathrm{B}$ virus and more than 50million people get infected every year, more than 2 million people die. There are 100-200million people with hepatitis C. ${ }^{1}$ Infection with hepatotropic viruses B, C, D is the cause of the development of chronic viral hepatitis $(\mathrm{CH})$, which are also among the most common liver diseases. ${ }^{2,3}$ Currently, there are more than 180million carriers of chronic hepatitis $\mathrm{C}$ and approximately 350 million carriers of chronic hepatitis B. ${ }^{4,5}$ Despite progress in modern medicine, difficulties remain in diagnosing $\mathrm{CH},{ }^{6}$ and adverse clinical manifestations of $\mathrm{CH}$ significantly worsen quality of life of patients and reduce its duration. ${ }^{6}$ Chronic hepatitis can lead to cirrhosis of the liver, liver failure and the development of hepatocellular liver cancer. ${ }^{1-3}$ Hepatocellular carcinoma is now becoming one him of the most common types of cancer in the world. It ranks sixth in incidence among all malignant neoplasms, making a significant contribution to the third leading cause of cancer mortality in the world..$^{7-9}$

The liver is the organ that is more often compared to other organs, is exposed to metastatic lesions, especially in cancer of the digestive tract. ${ }^{10}$ In pancreatic cancer, liver metastases occur in $50 \%$ of cases, gastric cancer metastasizes to the liver in $35 \%$ of cases, colorectal cancer from 20 to $50 \%$, breast cancer in $30 \%$, and esophageal cancer in $25 \% .^{10}$

In addition to viruses, hepatotropic poisons, drugs, and hypoxia result in damage to the liver tissue and the development of acute liver failure. ${ }^{8}$ Also a quite rare (from 0.1 to $3 \%$ ), but an actual problem is liver damage during pregnancy. The liver diseases caused by pregnancy are: acute fatty degeneration of the liver of pregnant women, HELLP syndrome, intrahepatic cholestasis of pregnant women, etc. ${ }^{11-13}$.
Hepatic complications that develop in acute diseases of the gastrointestinal tract, for example, in acute intestinal obstruction (AIO), occupy a particularly important place in urgent surgery. The overall mortality rate at an AIO remains high, ranging from $13 \%$ to $27 \%$, despite the improvement of methods for diagnosing and treating this pathology. ${ }^{14}$ More than $60 \%$ of all cases of AIO are small bowel obstruction. ${ }^{15-17}$ The most common form of acute small bowel obstruction is adhesive obstruction, which occurs mainly after laparotomies performed for various diseases of the abdominal organs (in $64-93 \%$ of cases). ${ }^{18,19}$ With the complication of acute small bowel obstruction with peritonitis and abdominal sepsis, the mortality rate increases to $43 \%$, and in the event of the syndrome of multiple organ failure and toxic-septic shock to $70-100 \% .^{18-20}$

Due to the fact that all the blood from unpaired organs of the abdominal cavity enters the liver through the portal vein, it is the first to take a toxic blow upon damage to the organs of the gastrointestinal tract. The flow of toxins and impaired blood circulation in the liver entail hepatocellular energy failure, manifested in the form of dystrophies of varying severity, as well as ischemia and necrosis of the liver tissue..$^{21,22}$

\section{Liver macroanatomy}

The liver is not only the largest organ in the human body, but also the largest gland. The mass of the liver is about $2-2.5 \%$ of the total body weight, or about $1.5 \mathrm{~kg}$ in an adult. The liver is located in the right hypochondrium, the dome-shaped upper surface of the liver is directly adjacent to the diaphragm, and only a relatively small part of the liver in an adult enters to the left of the median line and occupies the region of the epigastrium and left hypochondrium. ${ }^{23}$ Outside, the liver is covered with a visceral leaf of the peritoneum, and only a small area on the back of its surface is not covered by the peritoneum, forming an extraperitoneal field. The thin dense fibrous sheath under the peritoneum forms its own liver capsule (Glisson capsule).

Anatomically, human liver have two lobes: a large right and a smaller left lobe, separated from each other on the diaphragmatic surface by the crescent ligament, and on the visceral surface in front - by the groove of the round ligament of the liver, and behind the venous ligament ${ }^{24}$ the liver has 2 more lobes: square and caudate. The square lobe is bounded by the slit of the round ligament on the left; 
the fossa of the gallbladder on the right, behind it is limited by the gate of the liver. The caudate lobe is bounded by a slit of the venous ligament on the left, the groove of the inferior vena cava on the right, and the gate of the liver in front. The thickness of the liver decreases towards the left lobe..$^{25}$

According to the international anatomical nomenclature of 1980, in the liver, there are visceral and diaphragmatic surfaces, which are separated by the lower edge of the liver. ${ }^{23-25}$ The diaphragmatic surface (upper, anterior-upper) is convex and adjacent to the diaphragm, the visceral surface (lower) is facing down and back, there is a series of indentations from other abdominal organs: gastric, esophageal, duodenal, renal, adrenal gland depressure and colon intestinal depressure. ${ }^{26}$

The upper limit of the liver corresponds to the projection of the right dome of the diaphragm. On the middle clavicular line on the right, it is located at level $\mathrm{V}$ of the intercostal space. The lower border of the liver to the right along the mid-axillary line is determined at the level of the tenth intercostal space, then the lower border of the liver passes to the left without leaving the edge of the right costal arch. From the right middle clavicular line, the lower boundary goes from right to left and up. In the anterior midline, the lower border of the liver passes in the middle of the distance between the xiphoid process and the navel. At level $\mathrm{V}$ of the intercostal space, along the left breast bone line, the lower border of the liver connects to the upper border of the liver (Figure 1). ${ }^{23-25}$

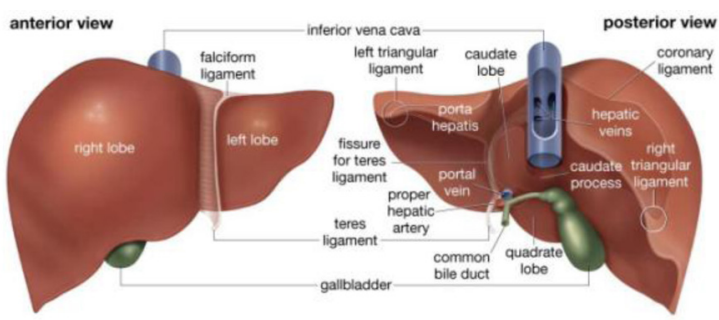

Figure I Liver macroanatomy.

\section{Segmental structure of the liver}

Speaking about the segmental structure of the liver, it is necessary to indicate that there are two options for dividing the liver into segments according to the "portal" and "caval" signs. The segmental structure of the liver was first described by a French surgeon and anatomist Claude Couinaud (C. Couinaud). ${ }^{25,26}$ In 1957, in the book "Le Foie: Etudes Anatomiques et Chirurgicales", Claude Couinaud noted the inconsistency of the internal structure of the blood and biliary tracts with external signs of liver division and suggested dividing the liver into segments, considering the branching of the portal vein system (according to the portal type). Due to the fact that the architectonics of the location of the branches of the portal vein, the hepatic artery and the bile ducts are identical, dividing it into segments according to the portal feature is anatomically more reasonable and more convenient in surgical practice. According to this classification, the liver is divided into 2 lobes (right and left), 5 sectors and 8 segments. ${ }^{27-31}$ Liver segments are relatively isolated sections of the liver parenchyma within the blood vessels of the liver - the portal vein branches, and hepatic artery of the third order ${ }^{28,29}$ between which there are small vascular gaps. The part of the liver that is supplied by the branch of the portal vein and the branch of the proper second-order hepatic artery is called the sector (Figure 2).

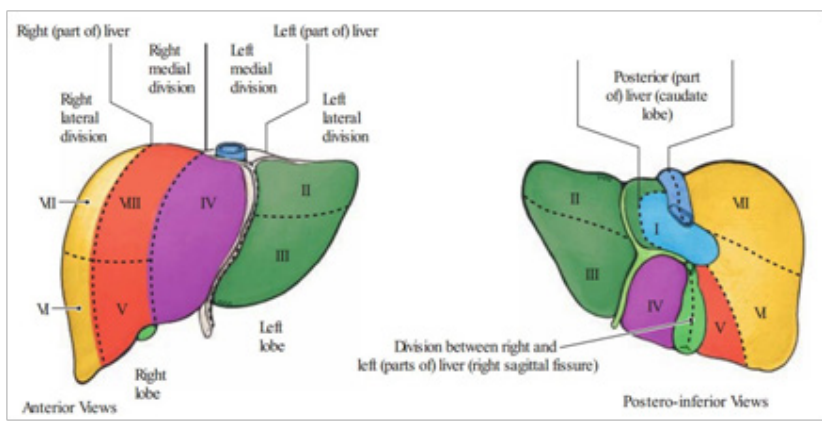

Figure 2 Segmental structure of the liver.

Couinaud 's work was of great importance for hepatobiliary surgery, since it became possible to perform organ-preserving complex liver resections, minimizing blood loss and the number of postoperative complications.

On the basis of the segmental structure of the liver and the branching of the portal vein, the functional division of the liver into lobes was performed. In 1898, in his works, J.Cantlie described in detail the true morphological boundary between the right and left lobes of the liver, proving that it is not at the level of the coronary ligament, but is determined by the sagittal furrow or line, which is now called Rex-Canllie, ${ }^{32,33}$ which anteriorly expands and forms the hole of the gallbladder, and posteriorly forms the sulcus of the inferior vena cava.

The numbering of the segments on the visceral surface of the liver in a clockwise direction starts from the furrow of the inferior vena cava. Thus, the first to the fourth segments is located in the left lobe, and in the right - from the fifth to the eighth segments. The first liver segment includes the caudate lobe of the liver and corresponds to the left dorsal sector (first sector). ${ }^{1,12,16,34}$ The second liver segment corresponds to the left lateral sector (second sector). The third segment and the fourth segment, formed by the square lobe of the liver, occupy the anterior part of the left lobe and the strip-like area on the diaphragmatic surface of the liver. Both segments belong to the left paramedian sector (third sector). The fifth and eighth segments belong to the right paramedian sector, which is the liver parenchyma bordering the left lobe of the liver along the sagittal groove (fourth sector). The lateral part of the right lobe of the liver consists of two segments - the sixth, located in front, and the seventh, occupying the posterior-lateral part of the diaphragmatic surface of the right lobe, and belongs to the right lateral sector (fifth sector)..$^{27,28,30,35}$

The blood from the liver flows into the inferior vena cava along the three hepatic veins: the upper right, middle and left main veins. The liver is divided according to the "caval" system according to the three main hepatic veins draining strictly isolated liver sections, ${ }^{26}$ but the segmental structure of the liver according to the "caval" type has not found wide application in practice. ${ }^{27,31}$

\section{Liver microanatomy}

The main functional unit of the liver is the hepatic lobule, which is a structure of a cylindrical hexagonal shape with a length of several millimeters, the diameter of the lobule varies from 0.8 to $2 \mathrm{~mm}$. Human liver consists of 50,000-100,000 individual lobules. The liver lobule slice is located around the central vein, which opens into the hepatic vein, which, in turn, is a branch of the inferior vena cava basin. 
Portal tracts (interlobular artery, interlobular vein, and interlobular bile duct) are located at the corners of the hexagonal lobule. Interlobular connective tissue in humans is normally poorly developed, the lobules do not have clear boundaries, unlike some animals in which it is well developed (pig, raccoon). In the parenchyma of the hepatic lobes, radially located in relation to the central vein are hepatic beams, consisting of two rows of hepatic hepatocyte cells, between which are the bile duct, which is the initial section of the biliary tract. Between the beams are sinusoids that collect blood from the interlobular arteries and veins and bring it into the central veins. ${ }^{36}$ Many small interlobular arterioles carry blood directly into the hepatic sinusoidal capillaries, with the most frequent option being flowing into the sinusoidal capillaries in the area $1 / 3$ of the distance from the interlobular septum to the central vein (Figure 3).

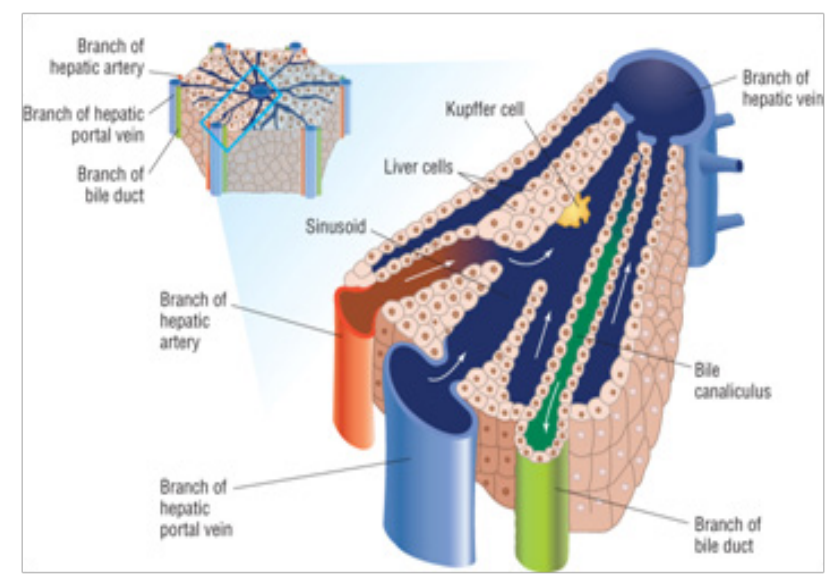

Figure 3 Liver lobule.

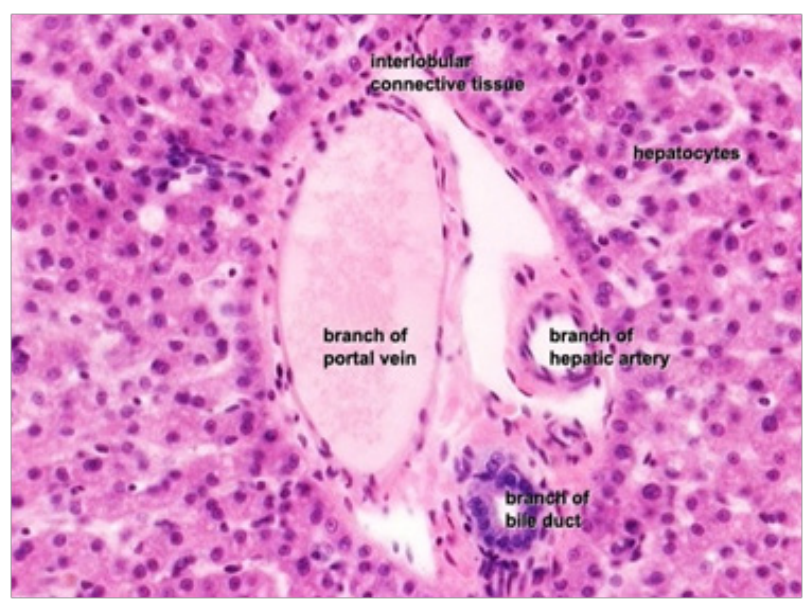

Figure 4 Histology of the portal tract.

The wall of sinusoids is separated from hepatocytes by the perisinusoidal space or the Disse space. Sinusoidal capillaries are lined with endothelial cells and Kupffer cells. The endothelial lining of sinusoids is lamellar and fenestrated, and therefore metabolism occurs unhindered between blood and hepatocytes.

Hepatocytes are polygonal in shape, ranging in size from 25 to 40microns with a pronounced eosinophilic cytoplasm and a centrally located nucleus. ${ }^{36}$ The nuclei of hepatocytes are round or oval and may contain glycogen, which is often detected at a young age, or in diseases such as diabetes and Wilson's disease. ${ }^{36}$

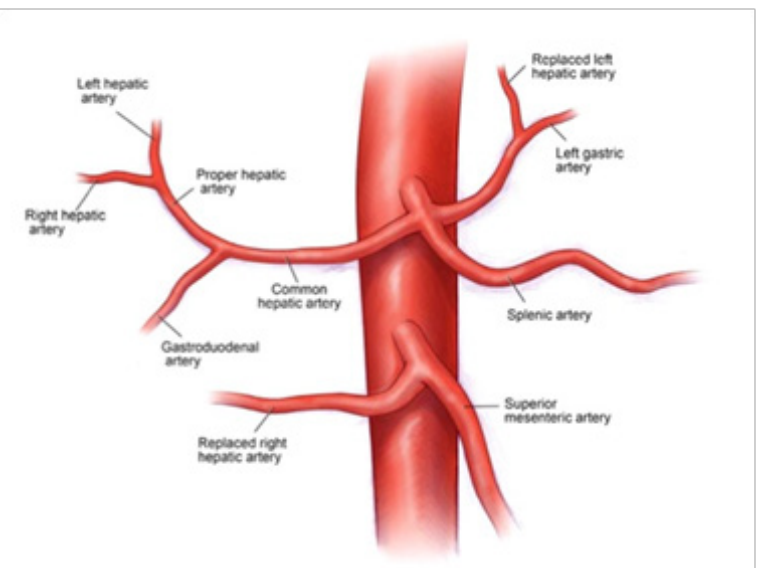

Figure 5 Arterial supplies to liver and aberrant arterial anatomy.

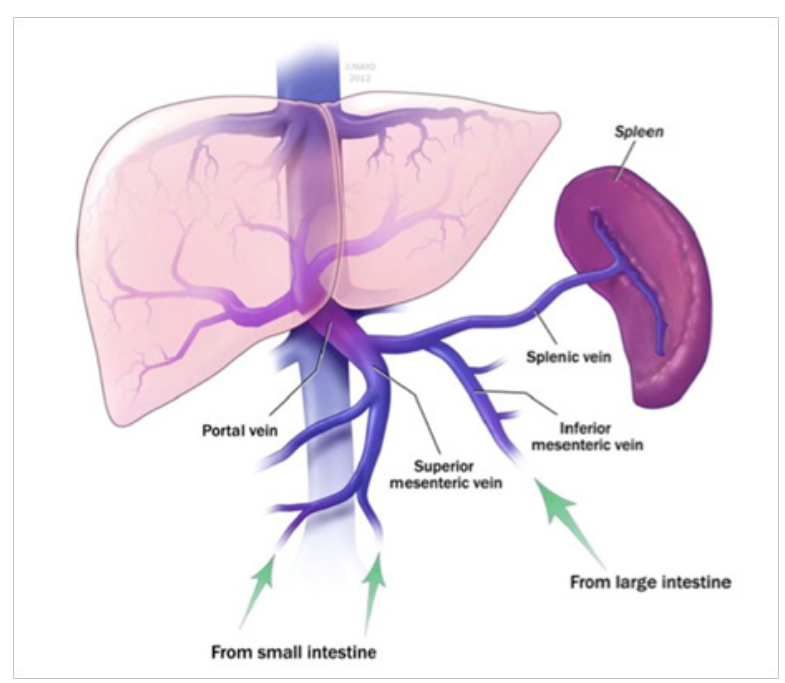

Figure 6 Portal venous drainage.

\section{Blood supply to the liver}

It is known that the liver is the richest blood supplying organ throughout the body. The mass of the liver is only $2-2.5 \%$ of body weight, but despite this, it receives $25 \%$ of the blood of the cardiac output, due to double blood supply (portal vein and hepatic artery). ${ }^{37}$

The blood enters the liver through two vessels, the portal vein carries blood from all unpaired abdominal organs (intestine, spleen, stomach, pancreas) enriched with substances that need to be processed and detoxified, and proper hepatic artery delivering blood rich in oxygen, so necessary for the maintenance of vital activity of hepatocytes and for their functional "job". ${ }^{38}$ Through these vessels, blood enters the sinusoids (common hepatic capillaries) - the microvasculature of the circulatory system of the liver, directly communicating with the hepatocytes, in which the metabolic processes and detoxification function of the liver are carried out. ${ }^{34,39,40}$ Some of them are equal to 1 micron. Behind this layer, located between it and the liver cells, there are narrow spaces called Disse spaces, or perisinusoidal spaces. Due to the presence of large pores in the endothelium of sinusoidal capillaries, substances from the plasma easily penetrate Disse spaces, and most of the plasma proteins easily diffuse into these spaces. The Disse spaces, in turn, communicate with the lymphatic vessels in the 
interlobular septa, so the fluid from these spaces has the ability to move into the intraorgan lymphatic system of the liver. Due to the fact that the pores of the sinusoidal capillaries of the liver are highly permeable and ensure the passage of not only solutions, but even proteins into Diss space, the lymph flowing from the liver contains proteins whose concentration is not much less than the concentration of proteins in the blood plasma. In addition, the extremely high permeability of the endothelium of sinusoidal capillaries of the liver contributes to the formation of large amounts of lymph. As a result, almost half of the total amount of lymph that forms in the body during rest is formed in the liver. After passing through the capillaries of the sinusoid, the blood enters the central vein of the liver lobules, which in turn is an inflow of the inferior vena cava basin.

Knowledge of options for the formation of liver arteries is of practical interest for surgeons performing surgical interventions in the hepatobiliary zone. ${ }^{41}$ In 1955, the American anatomist N.A. Michels published a book entitled «Blood supply and anatomy of the upper abdominal organs with a descriptive atlas», according to which 10 types of structure of the hepatic artery were isolated based on 200 autopsies. ${ }^{42}$ Over time, many authors generalized this classification, and even supplemented it with rare variants. ${ }^{42}$ This classification of the N. Michels blood supply options were developed for surgeons who performed interventions on the liver, stomach, bile ducts, and pancreas, and it was also developed taking into account the division of the liver on anatomical lobes. ${ }^{41,42}$

An important contribution to the study of the structure of the arteries of the liver made E.Z. Browne. ${ }^{19,40,43}$ For the first time E.Z. Browne in his work defined such terms as "common" and "proper" hepatic arteries. He designated the part of the hepatic artery extending from the celiac trunk to the place of the discharge of the gastroduodenal artery as a common hepatic artery. ${ }^{43}$ And the part of the artery going from the gastro-duodenal artery to the site of division into the right and left hepatic branches should be considered as proper hepatic artery.

In the recent literature over the past decade, many variants of the arterial blood supply to the liver have been described. ${ }^{44}$ The widespread introduction into practical public health of modern methods for the study of liver vessels allowed in vivo to study the arterial blood supply to the liver in large groups of patients. ${ }^{45}$ The proper hepatic artery passes to the left of the common bile duct and anterior to the portal vein in the left margin of the hepatoduodenal ligament. At the gate of the liver proper hepatic artery is divided into right and left hepatic artery. The right hepatic artery goes behind the common hepatic duct; in the Kahlo triangle, the cystic artery departs from it, which is the final branch, and therefore with its atherosclerotic occlusion, the development of stoneless destructive cholecystitis is possible. The length of the proper hepatic artery varies from 0.5 to $3 \mathrm{~cm}$, and its diameter is from 3 to $6 \mathrm{~mm} .{ }^{41,42}$ There are arteries that do not depart from proper hepatic artery, but at the same time take part in the blood supply to the liver, they are called aberrant or accessory. ${ }^{43,44}$

In the 18th century, the history of studying the anatomy of both the portal vein and its system began. ${ }^{39}$ The portal vein forms when the superior mesenteric and splenic veins merge, behind the pancreatic head. ${ }^{39}$ Up to $90 \%$ of the blood enters the liver exactly through the portal vein. ${ }^{39}$ Per minute, about 2.5 liters of blood flow through the portal vein system, which is $1 / 3$ of the total minute volume of human blood. The size of the portal vein in humans is $6-8 \mathrm{~cm}$ in length and
$1-1.5 \mathrm{~cm}$ in width. ${ }^{23}$ The length of the portal vein ranges from $2 \mathrm{~cm}$ to $8 \mathrm{~cm} .{ }^{39}$ Inside or at a distance of $1-1.5 \mathrm{~cm}$ from the gate of the liver, portal vein is divided into two branches: the wider right, joining the right lobe, and the narrower - left, going transversely from the liver gate to the left and carrying blood to the left and square lobes. ${ }^{41}$ Sometimes, before entering the liver, the portal vein is divided into three and sometimes four branches. ${ }^{39}$ Under normal conditions, most of the blood (70 to $75 \%$ ) from the trunk of the portal vein enters its right branch, and, respectively, in its right half, and its smaller part, only $25-30 \%$ - in the left branch, in the left part of liver. ${ }^{39,41}$ According to the literature, in the portal blood circulation system there is a decrease in the pressure of blood entering the capillaries from 110-120 to $10-15 \mathrm{~mm} \mathrm{Hg}$. v. ${ }^{41}$ In the portal vein, the pressure is $5-10 \mathrm{~mm} \mathrm{Hg}$. Art., and in the hepatic veins - $0-5 \mathrm{~mm} \mathrm{Hg}$. v. ${ }^{26}$ Due to the significant pressure difference in the initial and final sections of the portal bed, which is more than $100 \mathrm{~mm} \mathrm{Hg}$. Art., provided the translational movement of blood.$^{26}$ The portal vein is a valveless vessel that brings blood to the liver from the entire gastrointestinal tract and spleen, and the system of the hepatic veins drains blood into the inferior vena cava from the liver. ${ }^{23}$ The venous part of the vascular network of the liver is divided into 2 groups of anastomoses: hepatofugal (porto-caval) and hepatopetal (porto-portal). ${ }^{39}$ Porto-caval anastomoses connect the portal vein with the system of hollow veins, bypassing the liver, porto-portal anastomoses carry blood directly to the liver. Porto-portal anastomoses are numerous venous trunks lying in the ligaments of the liver, the largest port-portal veins are called accessory portal veins (Sappey veins). ${ }^{39}$

The outflow of blood from the hepatic lobules is provided by the central veins, which, when exiting the hepatic lobules, flow into the sublobular veins. ${ }^{23,39}$ Merging, the sublobular veins form hepatic veins, and they, in turn, are the efferent vascular system of the liver. ${ }^{23}$ The hepatic veins in the abdominal cavity are the last branches that the inferior vena cava takes. ${ }^{23,26}$ There are hepatic veins in the area of the furrow of the inferior vena cava and immediately fall into the inferior vena cava. ${ }^{23}$ In the classic case, 3 veins come out of the liver (right, middle and left), but according to the literature, the number of liver veins flowing into the inferior vena cava can reach 25 vessels. ${ }^{23,26}$. The right hepatic vein carries blood from the right lobe of the liver, the middle hepatic vein is from the square and caudate lobes, and the left hepatic vein carries blood from the left hepatic lobe. At the confluence of the hepatic veins into the inferior vena cava, its ampular expansion occurs. The area of the liver corresponding to the site of the exit of the hepatic veins and the passage of the inferior vena cava is called the second (caval) gate of the liver. ${ }^{25}$

\section{Innervation of the liver}

The innervation of the liver is carried out by the vagus nerves, the celiac plexus and the right phrenic nerve. In the lower esophagus, the right and left vagus nerves form the anterior and posterior vagus trunks, which are located on the corresponding surfaces of the lower esophagus? From the anterior vagus trunk, the hepatic branch departs, heading towards the left lobe and the gates of the liver as part of the hepato-gastric ligament. The posterior vagus trunk gives branches to the celiac plexus. The branches arising from this plexus are directed to the hepatoduodenal ligament along the common and proper hepatic artery, portal vein, as well as the bile ducts. In the hepatoduodenal ligament, branches coming from the celiac plexus, as well as the hepatic branch of the anterior vagus trunk, form the anterior and posterior hepatic plexuses, which are interconnected by numerous 
nerve branches. The anterior hepatic plexus is divided into two nerve bundles, which go along the right and left branches of the hepatic artery to the liver parenchyma. The posterior plexus is adjacent to the portal vein in the back and near the portal of the liver is located between the portal vein and the hepatic duct, and then its branches are sent to the liver.

The branches of the right phrenic nerve run along the inferior vena cava and penetrate the liver through the area nuda between the coronary ligaments of the liver. In function, the phrenic nerve (nervus phrenicus) is mixed, i.e. it consists of motor and sensitive branches. The phrenic nerve is the most powerful nerve in the cervical plexus. Through the motor branches, the nerve provides innervation to the muscles of the diaphragm, sensitive branches innervate the pleura, pericardium, diaphragm, and the peritoneum of the upper abdominal cavity, that is, including the peritoneum covering the liver and gall bladder. Consequently, irritation of the phrenic nerve in diseases of the liver and gallbladder is clinically manifested by difficulty breathing and the appearance of coughing movements, shortness of breath, hiccups, retching, pain in the chest, neck, supraclavicular fossa. Cholecysto-cardiac syndrome, a symptom complex characterized by a change in normal cardiac activity and manifested by cardialgia, arrhythmia, and cardiac conduction disturbance, may also occur.

Thus, the innervation of the liver is carried out by sympathetic (sympathetic hepatic plexus), parasympathetic (vagus nerve) fibers and peptidergic nerve fibers (which are an integral component of perivascular innervation), organized as afferent or efferent nerves with different origins and roles. ${ }^{3,23,46}$ All of them regulate blood flow through sinusoids, participate in the activation of Kupffer cells, in permeability of the endothelium of sinusoids, and in the functional activity of hepatocytes. ${ }^{46}$ Hepatocytes are the main cellular population of the liver, accounting for almost $80 \%$ of the total liver. The interaction between hepatocytes and nerve fibers occurs through many neurotransmitters and signaling pathways. Nerve fibers enter the liver through its gate along with the portal vein and hepatic artery. Nerve fibers enter the liver also through the small omentum and together with the hepatic veins. ${ }^{3,23}$ Sympathetic and parasympathetic nerves form two separate plexuses but interact with each other: the anterior plexus located around the hepatic artery and the posterior plexus located around the portal vein and the bile duct, then the nerve fibers are distributed in the liver parenchyma. In the human liver, nerve endings are located in the liver lobules, which consist of hepatocytes and nonparenchymal cells. Most nerve endings are located in Disse spaces, where they come into close contact with Kupffer cells, endothelial cells, and hepatocytes.

Sensory peptidergic nerve fibers (SPN) are a capsaicin-sensitive population of sensory fibers of the small neurons of the spinal ganglia, antidromically emitting neuropeptides-vasodilators (substance $\mathrm{P}$, calcitonin-related peptide - CRGR, neurokinin A), exercise local effector, including trophic function, are involved in the formation of neurogenic inflammation and pain syndromes. ${ }^{47}$

\section{Lymphatic system of the liver}

As noted above, the high permeability of the endothelium of sinusoidal capillaries of the liver contributes to the formation of large amounts of lymph and normally up to $50 \%$ of the lymph entering the thoracic lymphatic duct is formed in the liver. If the pressure in the vena cava is $10-15 \mathrm{~mm} \mathrm{Hg}$. Art., lymphatic drainage from the liver increases almost 20 times relative to the norm, and the "sweating" of fluid through the outer surface of the liver can become so large that it leads to the appearance of free fluid in the peritoneal cavity, which is called ascites. The blockage of blood flow through the portal system leads to the formation of high pressure in the capillaries of the portal system, including vessels of the gastrointestinal tract, with the development of edema of the intestinal wall with subsequent transudation of fluid through the serous membrane of the intestine into the abdominal cavity, which is the pathogenetic basis of ascites. If the pressure in the veins of the liver exceeds normal values by only 3-7 $\mathrm{mm} \mathrm{Hg}$. Art., it can cause the release of a significant amount of fluid in the lymphatic vessels of the liver and, in addition, her extravasation directly through the outer surface of the capsule of the liver into the peritoneal cavity. This liquid is almost no different in composition from blood plasma, except that it contains $80-90 \%$ more protein than plasma.

Lymphatic vessels of the liver are divided into superficial and deep. Deep vessels, in turn, are divided into ascending and descending. The ascending lymphatic vessels begin around the central veins, then converge into the large lymphatic vessels and travel along the hepatic veins to the posterior surface of the liver; together with the superficial vessels collecting lymph from the upper surface of the liver, they go to the lymph nodes of the chest cavity. On their way, these vessels pierce the diaphragm or pass through the slits and holes in it (hiatus aorticus, foramen v. Cavae, etc.). In the chest cavity, the lymphatic vessels of the liver flow into the intercostal, diaphragmatic (pericardial) and posterior mediastinal (esophageal) lymph nodes. ${ }^{48}$ Normally, the hepatic lymph nodes are not visible in various studies.

The descending lymphatic vessels run along the branches of the portal vein, hepatic artery and bile duct. Together with the superficial lymphatic vessels of the lower surface of the liver, they are directed to the hepatic lymph nodes located along the proper and common hepatic artery, left gastric artery, to the nodes of the omentum, as well as to the nodes surrounding the aorta and the inferior vena cava. The carrying lymphatic vessels of these nodes are sent to the celiac nodes surrounding the artery of the same name ${ }^{48}$ and then the lymph flows into the "milky" cistern (expansion of the thoracic lymphatic duct at its beginning). ${ }^{48-51}$ The lymphatic fluid through the thoracic lymphatic duct flows into the left subclavian vein and returns to the systemic circulation. $^{51}$

The superficial lymphatic network of the liver is located inside the Glisson capsule from the front and back of the liver. The lymph from the anterior lymphatic network of the liver primarily flows into the diaphragmatic lymph nodes, and then joins the mediastinal and internal lactic lymphatic networks. From the posterior superficial lymphatic network, the lymph is drained into the accompanying lymph nodes, cystic duct, common bile duct, hepatic artery and peripancreatic lymph nodes, as well as pericardial and celiac lymph nodes. The schemes of lymphatic drainage are of great importance in surgical practice for lymphadenectomy performed in cancer of the gallbladder, liver and pancreas..$^{23,49,51}$

\section{Discussion}

Anatomy is one of the oldest natural sciences. Its name comes from the Greek "anatemno", which means "dissecting", and arose at a time when the dissection of the corpses was the only source of knowledge about the human body. Since then, anatomy has come a long way and has been enriched by many research methods. Modern anatomy has and actively uses methods of radiation, electro-physical, modern 
optical and other methods of research for understanding the structure and variability of the body of a living person in all the diversity of its vital manifestations. The name of the anatomy now has a broader meaning than it was in the past.

Being a biological discipline, human anatomy is at the same time one of the foundations of medicine. Essentially, medical education begins with anatomy. The need for anatomical knowledge for physicians has been recognized and emphasized at all times. The founder of scientific anatomy, Andrei Vesalius, spoke of anatomy as a "pedestal and foundation of the medical art". One cannot but agree with the statement of the great Russian scientist and naturalist M. V. Lomonosov: "How can one talk about the human body without knowing either the addition of bones and joints to strengthen it, the union, the muscles for feeling, or the location of the viscera for the preparation of nutritious juices, no stretch of veins for the circulation of blood, nor other organs of this marvelous structure."

The importance of anatomy as one of the foundations of theoretical and practical medicine was well understood by the largest Russian scientists and doctors. At the beginning of the 19th century, the famous doctor and scientist E.O. Mukhin said: "A doctor is not an anatomist not only useless, but also harmful." And after almost $100 y e a r s$, AP Gubarev is one of the greatest Moscow clinicians, wrote no less figuratively: "Without anatomy, there is neither surgery nor therapy, but only signs and prejudices."

In our deep belief, based, among other things, on the personal experience of practical medical activity, the anatomical knowledge in medicine is comparable to the knowledge of the alphabet in literature, musical notation in music.

\section{Conclusion}

Detailed knowledge by practitioners and, in particular, by surgeons, both macroanatomy and liver microanatomy, contribute to the objectification of the choice of treatment tactics and, accordingly, to improve the results of treatment of both primary diseases and secondary lesions of the liver.

\section{Acknowledgments}

None.

\section{Conflict of interest}

The authors declare that there are no conflicts of interest.

\section{Funding}

None.

\section{References}

1. Modi AA, Liang TJ. Hepatitis C: A clinical review. Oral Dis 2008;14(1):10-14

2. Song JE, Kim DY. Diagnosis of hepatitis B. Ann Transl Med 2016;4(18):338

3. Tarrant D, Block J, McMahon B. Screening for Hepatitis B. The Journal for Nurse Practitioners. 2013;9(4):233-237.

4. Chang ML, Liaw YF. Hepatitis B flares in chronic hepatitis B Pathogenesis, natural course, and management. J Hepatol J Hepatol. 2014;61(6):1407-1417.
5. Hikspoors JP, Peeters MM, Kruepunga N, et al. Human liver segments: role of cryptic liver lobes and vascular physiology in the development of liver veins and left-right asymmetry. Sci Rep. 2017;7(1):17109.

6. Wu JF, Chang MH. Natural history of chronic hepatitis B virus infection from infancy to adult life -the mechanism of inflammation triggering and long-term impacts. J Biomed Sci. 2015;22:92.

7. Balogh J, Victor D 3rd, Asham EH, et al. Hepatocellular carcinoma: a review. J Hepatocell Carcinoma. 2016;3:41-53.

8. Pandit A, Mathew LG, Bavdekar A, et al. Hepatotropic viruses as etiological agents of acute liver failure and related-outcomes among children in India:a retrospective hospital-based study. BMC Res Notes. 2015;8:381

9. Waller LP, Deshpande V, Pyrsopoulos N. Hepatocellular carcinoma:A comprehensive review. World J Hepatol. 2015;7(26):2648-6263.

10. Gantsev KH, Ishmuratova RS, Atnabaev RD. Evalution of immediate and end results of surgery for gastro-intestinal tumors metastasing to the liver. Voprosy onkologii. 2008;54(2):188-191.

11. Lee NM, Brady CW. Liver disease in pregnancy. World J Gastroenterol. 2009;15(8):897-906

12. Mikolasevic I, Filipec-Kanizaj T, Jakopcic I, et al. Liver Disease during Pregnancy:A Challenging Clinical Issue. Med Sci Monit. 2018;24:4080-4090.

13. Westbrook RH, Dusheiko G, Williamson C. Pregnancy and liver disease. J Hepatol. 2016;64(4):933-945.

14. Magomedov MM. Optimization of complex treatment of acute intestinal obstruction. Vestnik novyh medicinskih tekhnologij. $2015 ; 4: 2-2$.

15. Lee H, Kim IK, Ju MK. Which patients with intestinal obstruction need surgery? The delta neutrophil index as an early predictive marker. Ann Surg Treat Res. 2017;93(5):272-276.

16. Markogiannakis H1, Messaris E, Dardamanis D, et al. Acute mechanical bowel obstruction:Clinical presentation, etiology, management and outcome. World J Gastroenterol. 2007;13(3):432-437.

17. Pujahari AK. Decision making in bowel obstruction:A Review. J Clin Diagn Res. 2016;10(11):PE07-PE12.

18. Dubrovshchik OI, Marmysh GG, Dovnar IS, et al. Adhesive ileus:tactics, treatment, prevention of recurrences. Journal of Grodno State Medical University. 20122(38):20-23.

19. Catena F, Saverio SD, Coccolini F, et al. Adhesive small bowel adhesions obstruction:Evolutions in diagnosis, management and prevention. World J Gastrointest Surg. 2016;27;8(3):222-231.

20. Ruler O, Boermeester MA. Surgical treatment of secondary peritonitis. A continuing problem. Chirurg. 2017;88(Suppl 1):1-6.

21. Guicciardi ME, Malhi H, Mott JL, et al. Apoptosis and Necrosis in the Liver. Compr Physiol. 2013;3(2):977-1010.

22. Waseem N, Chen PH. Hypoxic Hepatitis: A Review and Clinical Update. J Clin Transl Hepatol. 2016;4(3):263-268.

23. Abdel-Misih SR, Bloomston M. Liver Anatomy. Surg Clin North Am 2010;90(4):643-653.

24. Chaudhari HJ, Ravat MK, Vaniya VH, et al. Morphological study of human liver and its surgical importance. J Clin Diagn Res. 2017;11(6):AC09-AC12

25. Fasel J, Schenk A. Concepts for liver segment classification:neither old ones nor new ones, but a comprehensive one. J Clin Imaging Sci. 2013;3:48. 
26. Sibulesky L. Normal liver anatomy. Clin Liver Dis (Hoboken). 2013;2(Suppl 1):S1-S3.

27. Alghamdi T, Viebahn C, Justinger C, et al. Arterial Blood Supply of Liver Segment IV and Its Possible Surgical Consequences. Am J Transplant. 2017;17(4):1064-1070.

28. Couinaud C. The Liver: Anatomical and Surgical Studies. Paris, France: Masson;1957. p. 530.

29. Couinaud C. Liver anatomy: portal (and suprahepatic) or biliary segmentation. Dig Surg. 1999;16(6):459-467.

30. Chanwat R, Bunchaliew C, Tanompetsanga R, et al. How I do it: laparoscopic anatomical segment 8 resection. Ann Laparosc Endosc Surg. 2018;

31. Hikspoors JPJM, Peeters MMJP, Kruepunga N, et al. Human liver segments:role of cryptic liver lobes and vascular physiology in the development of liver veins and left-right asymmetry. Sci Rep. 2017;7(1):17109.

32. Sakamoto Y, Kokudo N, Kawaguchi Y, et al. Clinical anatomy of the liver: review of the 19th meeting of the japanese research society of clinical anatomy. Liver Cancer. 2017;6(2):146-160.

33. Yamamotoa Y, Oldhaferb KJ. Frontiers of Liver Surgery. Visc Med. 2017;33(6):405-406

34. Maronne G, Shah VH, Gracia-Sancho J. Sinusoidal communication in liver fibrosis and regeneration. J Hepatol. 2016;65(3):608-617.

35. Mishra SP, Tiwary SK, Mishra M, et al. An introduction of Tertiary Peritonitis. J. J Emerg Trauma Shock. 2014;7(2):121-123.

36. Krishna M. Microscopic Anatomy of the Liver. Clin Liver Dis. 2013;2(Suppl 1):S4-S7.

37. Prozorovsky KV, Pruchansky VS. Peculiarties of blood flow in the main portal vein vis-à-vis patterns of hepatic neoplasia. Vopr Onkol. 2008;54(4):498-500.

38. Eipel C, Abshagen K, Vollmar B. Regulation of hepatic blood flow: The hepatic arterial buffer response revisited. World J Gastroenterol. 2010;16(48):6046-6057.

39. Levenets SV. History of study of the anatomical variation of the portal vein (review of literature). Zagal'napatologija ta patologichna. fiziologija. 2014;9(2):5-11.
40. Fernandez-Iglesias A, Gracia-Sancho J. How to face chronic liver disease: the sinusoidal perspective. Front Med (Lausanne). 2017;4:7.

41. Catalano OA, Singh AH, Uppot RN, et al. Vascular and biliary variants in the liver: Implications for liver surgery. Radiographics. $2008 ; 28(2): 359-378$

42. Rummo OO, Korotkov SV, Scherba AE, et al. Hepatic artery anatomy: a clinical importance in the liver transplantation. Annaly Khirurgicheskoy Gepatologii. 2011;6(3):72-78.

43. Balakhnin PV, Tarazov PG, Polikarpov AA, et al. The Angiographic Evaluated of the Hepatic Arterial Anatomy Variations in 1511 Patients. Annals of HPB Surgery. 2004;9(2):1-10.

44. Qiao JL, Sun J, Li J, et al. Liver dual arterial blood supply maintains liver regeneration: Analysis of signaling pathways in rats. Mol Med Rep. 2018;17(1):979-987.

45. Ralls PW. Color doppler sonography of the hepatic artery and porta venous system. AJR Am J Roentgenol. 1990;155(3):517-525.

46. Mizuno K, Ueno Y. Autonomic Nervous System and the Live. AJR Am J Roentgenol. 1990;155(3):517-525.

47. Krupatkin AI. The role of sensory peptidergic nerve fibers and neurogenic inflammation in the development of posttraumatic complex regional pain syndrome. Zh Nevrol Psikhiatr Im $S$ S Korsakova. 2014;114(5):12-16.

48. Zhdanov DA. General anatomy and physiology of the lymphatic system. Leningrad: Medgiz;1952. p.336.

49. Pupulim L, Vilgrain V, Ronot M, et al. Hepatic lymphatics:anatomy and related diseases. Abdom Imaging. 2015;40(6):1997-2011.

50. Qiao JL, Sun J, Li J, et al. Liver dual arterial blood supply maintains liver regeneration:Analysis of signaling pathways in rats. Mol Med Rep. 2018;17(1):979-987.

51. Tanaka M, IwakiriY. The Hepatic Lymphatic Vascular System:Structure, Function, Markers, and Lymphangiogenesis. Cell Mol Gastroenterol Hepatol. 2016;2(6):733-749. 